\title{
Different Phenolic Extracts of Oil Palm Fruits and Caffeic Acid Prevent Palmitic Acid-Induced Lipotoxicity in HepG2 Cells via Improving Mitochondrial Function
}

\author{
Xiaojing Liu, ${ }^{1}$ Ou Wang, ${ }^{2}$ Jiexin Zhou, ${ }^{1}$ and Shengbao Cai ${ }^{1}{ }^{1}$ \\ ${ }^{1}$ Faculty of Agriculture and Food, Yunnan Institute of Food Safety, Kunming University of Science and Technology, Kunming, \\ Yunnan 650500, China \\ ${ }^{2}$ National Institute for Nutrition and Health, Chinese Center for Disease Control and Prevention, Beijing 100050, China
}

Correspondence should be addressed to Shengbao Cai; caikmust2013@163.com

Received 17 June 2020; Revised 10 July 2020; Accepted 14 July 2020; Published 3 August 2020

Academic Editor: Quancai Sun

Copyright (c) 2020 Xiaojing Liu et al. This is an open access article distributed under the Creative Commons Attribution License, which permits unrestricted use, distribution, and reproduction in any medium, provided the original work is properly cited.

Oil palm (Elaeis guineensis Jacq.) fruits are rich in antioxidative phenolic compounds, which exert various health improving effects. This study aimed to comparatively study the protective effects of different phenolic fractions from the fruits treated with or without ultrahigh pressure (UHP) and their most abundant phenolic substance, caffeic acid, against the palmitic acid-induced lipotoxicity in HepG2 cells and to clarify the potential mechanisms. Results showed that the TG accumulation, intracellular reactive oxygen species content, cell apoptosis, and mitochondrial membrane potential depolarization were relieved by the administration of those phenolic fractions and caffeic acid. The protection of insoluble-bound (IB) phenolic fraction was the best, and UHP treatment significantly enhanced its protective effect on lipotoxicity. The lipotoxicity preventive effect may be achieved by alleviating intracellular oxidative stress, downregulating the expression of P-P38, COX-2, and iNOS to relieve inflammation, and downregulating Bax and cytochrome $\mathrm{C}$ and upregulating $\mathrm{Bcl}-2$ to suppress the mitochondrial-mediated apoptosis. Therefore, the oil palm fruits phenolic fractions, especially the IB phenolic fraction, might be utilized as dietary antioxidants to relieve the adverse effects of a high-fat diet on the body, and UHP treatment is a potential method to increase this bioactivity.

\section{Introduction}

High-fat diet consumption is very common in modern society, which could easily lead to high level-free fatty acid (FFA) in the body circulation system and excessive fat deposition in organs [1]. Excessive intake of FFA accompanied by large amounts of fat (mainly triglyceride, TG) accumulated in cells of different organs can lead to chronic injury and dysfunction of those organs, which is defined as lipotoxicity $[2,3]$. The lipotoxicity has been proved to be closely related to many metabolic dysfunction diseases, such as hyperlipidemia, fatty liver, and diabetes $[4,5]$. For instance, when hepatocytes ingest a large amount of FFA, on the one hand, the FFA is stored as triglycerides in hepatocytes, resulting in hepatic steatosis [6]; on the other hand, the FFA is oxidized, leading to many reactive oxygen species
(ROS) production and thereby damaging hepatocytes; and these will lead to the occurrence of fatty liver or/and steatohepatitis $[6,7]$. Some natural compounds in dietary plants with a good free radical scavenging activity can prevent oxidative stress-induced liver injury [8].

Among the FFAs, palmitic acid (PA) is abundant in the human body and widely distributed in daily diet. Based on these, PA has been used to induce lipotoxicity in the in vitro studies, especially in cells model, such as hepatocytes, islet $\beta$-cells, and endothelial cells [9]. Inflammation response, endoplasmic reticulum stress, mitochondrial damage, and cell apoptosis were proved to be involved in PA induced lipotoxicity $[2,5,9,10]$. During the excessive PA intake, massive reactive oxygen species (ROS) were generated along with the lipid peroxidation, which probably stimulates inflammation response and cell apoptosis, and eventually leads 
to physiological dysfunction [7, 11]. Previous researches reported that ROS could cause an inflammatory response by the activation of the P38 mitogen activated protein kinases (MAPK) pathway and regulate the secretion of inflammatory cytokines, such as cyclooxygenase-2 (COX-2) and inducible nitric oxide synthase (iNOS) [12, 13]. Besides, under the stimulation of ROS and inflammation, cell apoptosis could be activated, and the mitochondrial pathway was involved in the possible mechanism $[11,14]$. In the mitochondrial pathway, the B cell lymphoma-2 (Bcl-2) family could interact with mitochondria members to disrupt mitochondrial membrane potential (MMP) and mitochondrial permeability. Bax, a proapoptosis of the Bcl-2 family, could be activated by inflammation and then bind with the mitochondrial membrane to open the permeability transition channels. Meanwhile, the antiapoptosis Bcl-2 could be suppressed $[15,16]$. In a case like this, cell apoptosis was activated and markers of apoptosis were released, like cytochrome $\mathrm{C}$, a protein that has been widely accepted as the marker of apoptosis [17].

Since the negative effect of high FFAs consumption on health has been widely proved, and no effective clinical drug is available, it is necessary to explore bioactive compounds from food materials to decrease the damage of FFAs. Among the numerous natural compounds, antioxidative phenolic compounds showed effectively protective effects against lipotoxicity induced by FFAs, such as PA [18]. In our previous research, we reported that oil palm fruit (OPF) was rich in phenolic compounds, especially caffeic acid, and exhibited good antioxidant activity in vitro; moreover, we found that ultrahigh pressure (UHP) treatment increased the phenolic compounds bioaccessibility and the antioxidant of OPF [19]. Therefore, we hypothesized that OPF and its main phenolic compound, caffeic acid, might effectively prevent FFA-induced lipotoxicity and OPF treated by UHP may have a better effect, which has not been investigated. In the present study, the free (F), esterified (E) and insolublebound (IB) phenolic fractions were extracted from UHPtreated or nontreated OPF. Their protective effects and the caffeic acid on PA-induced lipotoxicity would be comparatively investigated by the analyses of triglyceride (TG) accumulation, intracellular ROS scavenging ability, cell apoptosis, and MMP. Moreover, the expression levels of several key markers in important signaling pathways of inflammation and apoptosis were also testified by western blot to illuminate the potential mechanism. The results of this study might provide scientific information for the devolvement of OPF product as a functional food to prevent or alleviate FFA-induced lipotoxicity.

\section{Materials and Methods}

2.1. Materials and Chemical Reagent. The OPF was obtained from a local market in Haikou city (Hainan province, China). The human hepatocellular carcinoma cell line (HepG2) was obtained from Kunming cell bank (Chinese Academy of Sciences, China). Fetal bovine serum (FBS), penicillin, streptomycin, and Dulbecco's modified Eagle's medium (DMEM) were supplied by Gibco (Grand Island,
NY). Palmitic acid, methylthizol-2-yl-2,5- diphenyl tetrazolium bromide (MTT), and $2^{\prime}, 7^{\prime}$-dichlorofluorescin diacetate (DCFH-DA) were purchased from Sigma-Aldrich (Shanghai, China). Bovine serum albumin (BSA) was purchased from BioDee Biotech Co., Ltd. (Beijing, China). Annexin V-FITC/PI apoptosis kit was obtained from Beijing 4A Biotech Co., Ltd. (Beijing, China). Cell lysis buffer, TG assay kit, BCA protein assay kit, and mitochondrial membrane potential assay kit (JC-1) were purchased from Shanghai Beyotime Co., Ltd. (Shanghai, China). Caffeic acid standards were supplied by Chengdu Must Bio-Technology Co., Ltd. (Chengdu, China). All antibodies were procured from Abcam (Cambridge, UK), and other reagents used were of analytical grade.

\subsection{Preparation and Analysis of Different Phenolic Fractions} from OPF. The UHP pretreatment of OPF and preparation of different phenolic fractions (F, E, and IB) were conducted as a previous report [19]. Briefly, chopped mesocarps were vacuum-sealed and then pretreated by a UHP equipment (HHP-600, Baotou Kefa High Pressure Technology Limited Company, Baotou, China) with $500 \mathrm{MPa}$ pressure for 10 min. Subsequently, the F, E, and IB phenolic fractions were extracted from OPF with or without a UHP pretreatment, respectively. The phenolic compositions of different fractions were analyzed by UHPLC-ESI-HRMS/MS in negative mode. As we reported previously, a total of 13 phenolic compounds were detected, including caffeic acid, catechin, gallic acid, protocatechuic acid and kaempferol, and caffeic acid was the predominant phenolic compound in all fractions [19].

2.3. PA/BSA Complex Solution Preparation. The PA/BSA complex solution was prepared according to the previous method with some modifications [20]. Briefly, PA was dissolved with $0.1 \mathrm{M} \mathrm{NaOH}$ into $100 \mathrm{mM}$ in a shaking water bath at $70^{\circ} \mathrm{C}$ for $30 \mathrm{mins}$. Then, $50 \mu \mathrm{L}$ PA solution was mixed with $950 \mu \mathrm{L}$ BSA $(10 \%)$ and incubated in a water bath at $70^{\circ} \mathrm{C}$ for six hours. Finally, the PA/BSA complex solution was sterile filtered with $0.22 \mu \mathrm{m}$ membrane filter and stored at $-20^{\circ} \mathrm{C}$ as stock. DMEM was used to dilute the stock into appropriate concentration for the experiment, and the same concentration of $\mathrm{BSA} / \mathrm{NaOH}$ complex was used as a vehicle in each assay.

2.4. Determination of PA and Sample Cytotoxicity. The HepG2 cell was cultured in DMEM supplemented with $10 \%$ FBS and 5\% penicillin-streptomycin at an atmosphere of 5\% $\mathrm{CO}_{2}$ at $37^{\circ} \mathrm{C}$. The culture medium was changed every two days. Cells grown up to $90 \%$ confluence were used for subsequent experiments. MTT method was conducted as our previous report to evaluate the cytotoxicity of PA and different phenolic fractions on HepG2 [19]. In brief, after being seeded in a plate $\left(1 \times 10^{5} / \mathrm{mL}\right)$ with a 24 -hour incubation, cells were stimulated with PA/BSA complex solution at the concentrations of $0.1 \mathrm{mM}, 0.2 \mathrm{mM}, 0.3 \mathrm{mM}, 0.4 \mathrm{mM}$, and $0.5 \mathrm{mM}$ or different concentrations of the sample ranging 
from $40.0 \mu \mathrm{g} / \mathrm{mL}$ to $200.0 \mu \mathrm{g} / \mathrm{mL}$. For another 16-hour (PA) or 24-hour (sample) incubation, the treatment medium was removed, and cells were washed twice with PBS solution. Then, $0.5 \mathrm{mg} / \mathrm{mL}$ MTT solution was added, and then cells were incubated for another four hours. Thereafter, MTT solution was discarded and $150 \mu \mathrm{L}$ of DMSO was added into each well to completely solubilize the formazan. The absorbance of each well was recorded by SpectraMax M5 microplate reader (Molecular Device, USA) at $570 \mathrm{~nm}$. The cells treated with DMEM were used as the corresponding control group. The control group was considered as 100\% viability. Each group was set with six repeating wells, and each experiment was conducted three times.

2.5. Measurement of Intracellular TG Level in PA-Induced HepG2 Cells. The intracellular TG level in PA-induced HepG2 cells was determined according to a published method with some modifications [9]. Briefly, HepG2 cells were seeded in a six-well plate at a density of $1 \times 10^{5} / \mathrm{mL}$ with $2 \mathrm{~mL}$ per well. After incubation for 24 hours, $200 \mu \mathrm{L}$ of F, E, IB phenolic fractions or caffeic acid dissolved in DMEM with $120.0 \mu \mathrm{g} / \mathrm{mL}$ were separately added into the corresponding well for another 24-hour incubation, while cells in Control (CK), BSA, and PA groups were still cultured with DMEM. Thereafter, HepG2 cells in sample and PA groups were exposed to $0.3 \mathrm{mM}$ of PA/BSA complex solution for 16 hours, and cells in BSA and CK groups were incubated with the corresponding concentration of $\mathrm{BSA} / \mathrm{NaOH}$ complex solution and DMEM, respectively. Then, HepG2 cells were washed twice with PBS, lysed with lysis buffer $(200 \mu \mathrm{L}$ per well), homogenized with ultrasonic cell crusher (Scientz-II D, Ningbo Scientz Biotech Co., Ltd. Ningbo, China) and centrifuged at $4^{\circ} \mathrm{C}$ with $6000 \mathrm{~g}$ for five minutes. The supernatant was collected for determining protein and TG contents with BCA protein assay kit and TG assay kit, respectively, according to the manufacturer's instructions. Each group was set with three repeating wells, and each experiment was conducted three times.

2.6. Inhibition of Intracellular ROS. HepG2 cells were cultured and treated as mentioned in Section 2.5. After incubation, cells in all groups were washed with PBS for three times, and then harvested and labeled with DCFH-DA. Thereafter, FBS-free medium was used to wash the uncombined dyes and resuspend the cells. The fluorescence was detected with a flow cytometer (Guava easy Cyte 6-2L, Millipore, Billerica, USA) as the previous report [19]. The relative amount of intracellular ROS in each group was calculated by taking the CK group as $100 \%$ for reference.

2.7. Analysis of Apoptosis. HepG2 cells were treated as mentioned in Section 2.5. After 16-hour incubation in PA/ BSA complex solution, the apoptosis of cells was analyzed with Annexin V-FITC/PI apoptosis kit [19]. Briefly, harvested cells were washed with $\mathrm{PBS}$, and then $1.0 \mathrm{mg} / \mathrm{mL}$ Annexin V-FITC and $20.0 \mu \mathrm{g} / \mathrm{mL}$ PI were successively applied to stain the cells. Thereafter, the apoptosis rate was detected by a Guava easy Cyte 6-2L flow cytometer as the previous report.

2.8. Analysis of Cellular MMP. HepG2 cells were treated as mentioned in Section 2.5. After 16-hour incubation in PA/ BSA complex solution, the MMP of HepG2 cells was analyzed by a mitochondrial membrane potential assay kit (JC1) based on the instructions of the manufacturer. After harvest, cells were labeled with $1 \mathrm{~mL}$ JC-1 (1X) and incubated at $37^{\circ} \mathrm{C}$. About 30 mins later, JC-1 (1X) was removed, and $1 \mathrm{~mL}$ ice-cold JC-1 buffer (1X) was added to wash the cells twice. Then cells were resuspended with $1 \mathrm{~mL}$ FBS-free medium and determined with a Guava easy Cyte 6-2L flow cytometer within 30 mins. The mitochondrial membrane depolarization level was reflected by the ratio of red fluorescence intensity to green fluorescence intensity.

2.9. Western Blot Analysis. The cell treatments were as the same as mentioned in Section 2.5. The target proteins were separated by $12 \%$ sodium dodecyl sulfate-polyacrylamide gel (SDS-PAGE) electrophoresis, electrotransferred to the nitrocellulose membrane (Gelman Laboratory, Ann Arbor, USA), and blocked with 5\% BSA for $1 \mathrm{~h}$. Then, the membrane was incubated with the corresponding primary antibody overnight at $4^{\circ} \mathrm{C}$. After being washed three times with TBS-Tween $20(0.1 \%, v / v)$, the membrane was incubated with secondary antibody for another $1 \mathrm{~h}$ at room temperature and washed with TBS-Tween 20 again. Thereafter, the image of each protein was captured by a VILBER Fusion FX7 imaging system (Vilber Lourmat, Marne-la-Vallée, France) with enhanced chemiluminescent detection reagent (Millipore, Billerica, MA, USA).

2.10. Statistical Analysis. All experiments were conducted at least three times. Data were presented as mean \pm standard deviation (SD) and analyzed with Origin 8.5 software (OriginLab, Northampton, MA, USA). The significant differences between groups were analyzed with One-Way ANOVA followed by Tukey's test $(p<0.05)$.

\section{Results and Discussion}

3.1. Phenolic Fractions and Caffeic Acid Attenuated the PAInduced TG Accumulation. In the present work, the appropriate concentration of PA used for inducing lipotoxicity in HepG2 cells was investigated by MTT assay. As shown in Figure 1(a), the cell viability decreased gradually with the increase of PA concentration. In order to successfully establish the lipotoxicity cell model for subsequent research, the cell viability needs to be maintained at a certain extent. In this work, the cell viability was about $70 \%$ and $60 \%$ at $0.3 \mathrm{mM}$ and $0.4 \mathrm{mM}$ of PA, respectively, which indicated that cells suffered a moderate lipotoxicity. Therefore, both $0.3 \mathrm{mM}$ and $0.4 \mathrm{mM}$ of PA can be used to establish the lipotoxicity cell model, and in this work, $0.3 \mathrm{mM}$ of PA was applied. Moreover, the MTT assay suggested that all samples showed no toxicity to HepG2 cells at the concentrations up 


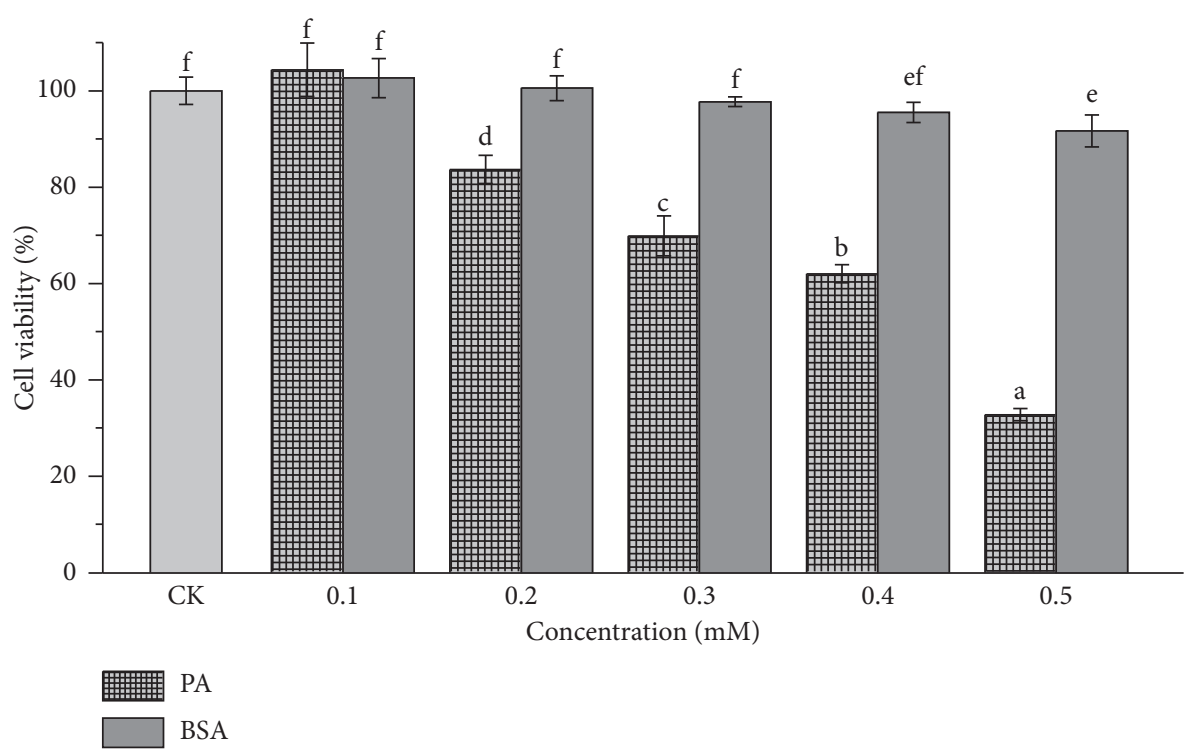

(a)

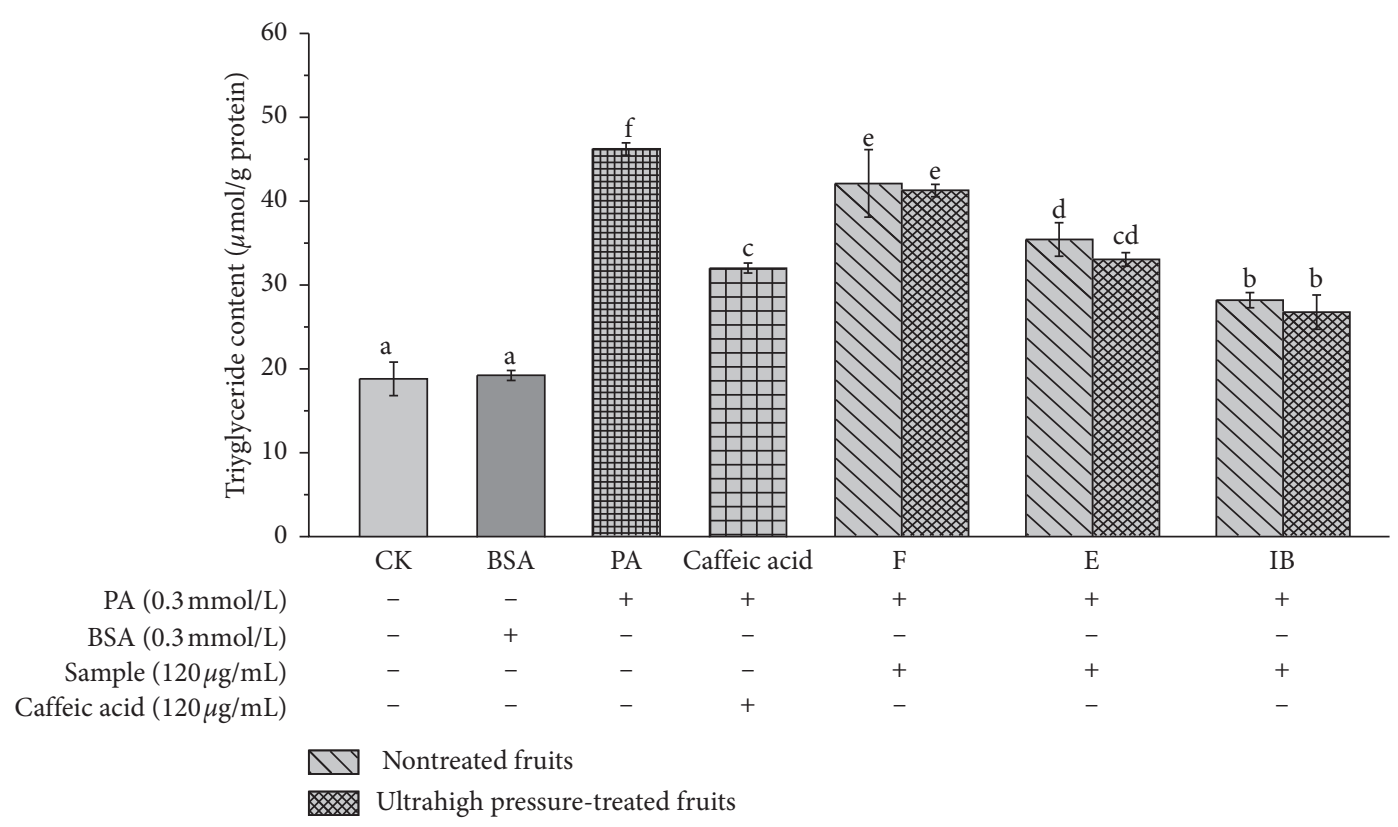

(b)

Figure 1: (a) The MMT result of PA, (b) the effects of phenolic fractions from oil palm fruits with or without ultrahigh pressure treatment and caffeic acid on PA-induced TG accumulation. Data are expressed as mean $\pm \mathrm{SD}(n=3)$. Mean values with different letters are significantly different $(p<0.05)$. CK: control group; BSA: BSA/NaOH complex solution; PA: PA/BSA complex solution; "F" free phenolic fraction; "E" esterified phenolic fraction; "IB" insoluble-bound phenolic fraction.

to $120 \mu \mathrm{g} / \mathrm{mL}$, which was consistent with our previous report [19].

Long term and excessive fat intake are easy to result in lipotoxicity to cause fat deposition and eventually lead to obesity, fatty liver disease, or other chronic diseases [21]. Thus, the effects of different phenolic fractions from OPF on TG deposition in HepG2 cells stimulated by PA were presented in Figure 1(b). The TG content of control group and BSA group was similar $(p>0.05)$, with values of $18.82 \pm 2.00$ $\mu \mathrm{moL} / \mathrm{g}$ of protein and $19.32 \pm 0.61 \mu \mathrm{moL} / \mathrm{g}$ of protein, respectively, while the TG level in PA group was about
$46.18 \pm 2.05 \mu \mathrm{moL} / \mathrm{g}$ of protein, which was approximately two times more than that in the CK group or BSA group $(p<0.05)$ and indicated significant fat deposition in hepatic cells. When treated with different phenolic fractions of OPF, all groups showed significantly lower TG levels than that of the PA group $(p<0.05)$, indicating significant effects on inhibition of TG deposition. Regardless of UHP treatment, the IB fraction exhibited the best inhibition effect among the three phenolic fractions on TG accumulation. With the administration of IB fraction, the intracellular TG level was $28.16 \pm 0.90 \mu \mathrm{moL} / \mathrm{g}$ of protein in nontreated fruit group, 

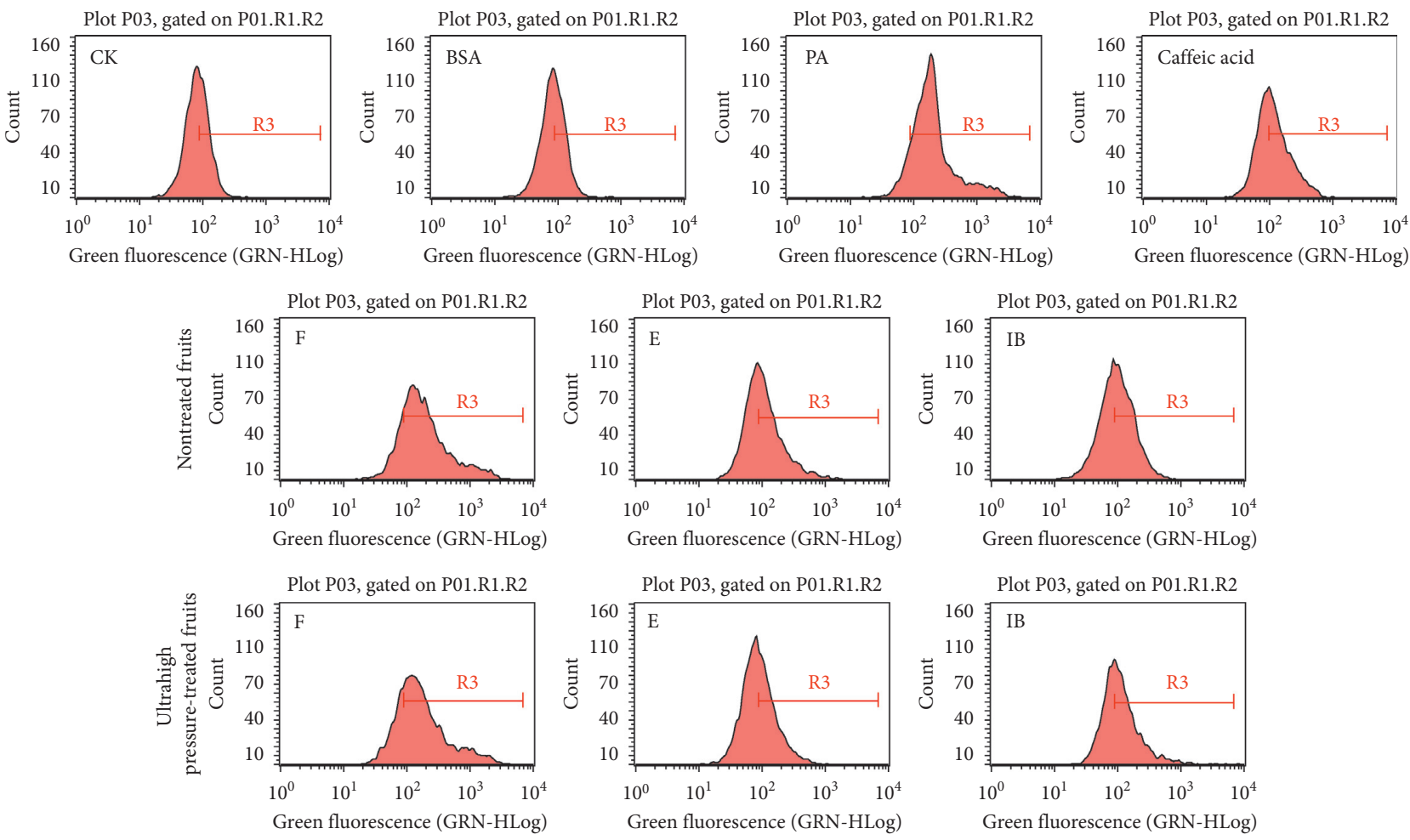

(a)

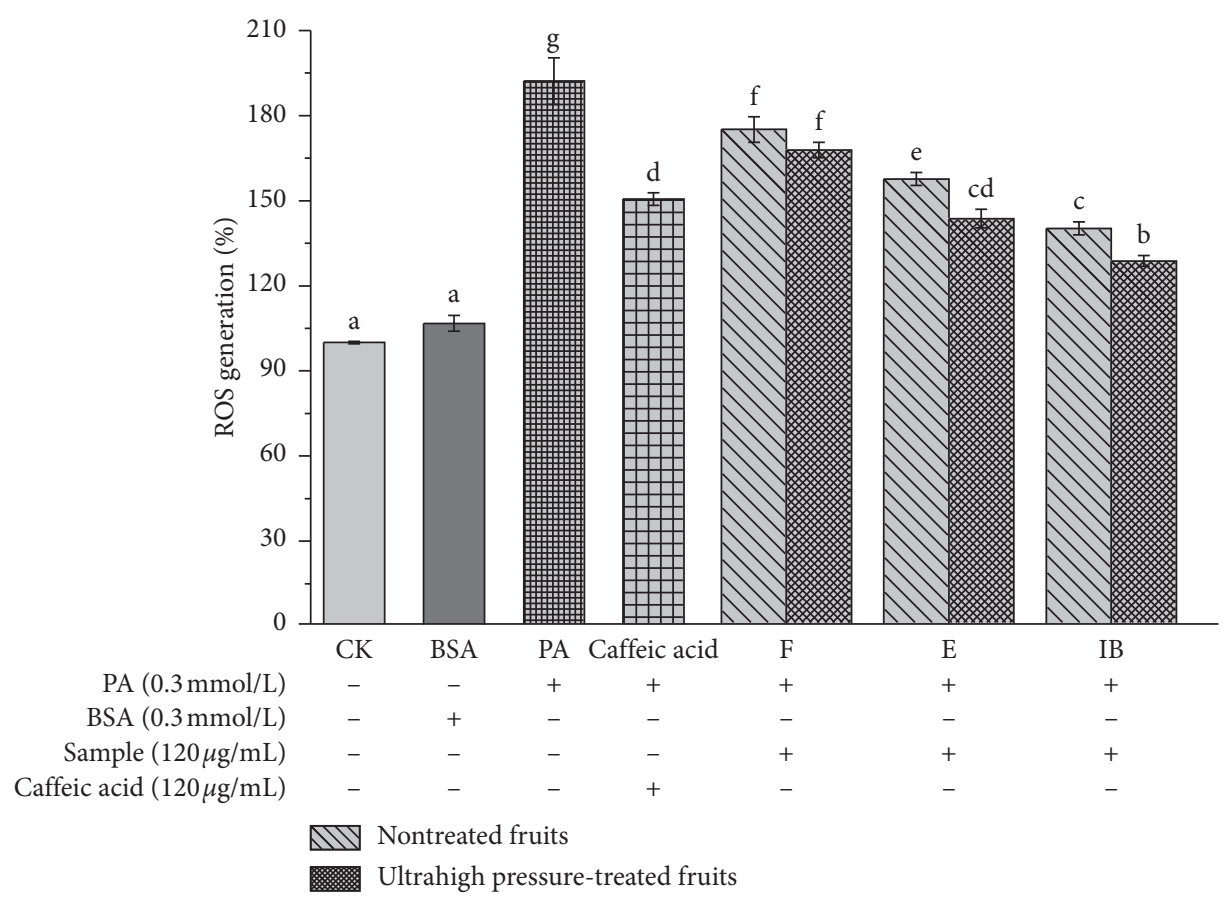

(b)

FIGURE 2: The inhibitory effects of phenolic fractions from oil palm fruits with or without ultrahigh pressure treatment and caffeic acid on PA-induced ROS generation: flow cytometry analysis (a) and the relative amount of ROS of different groups (b). Data are expressed as mean \pm SD $(n=3)$. In Figure 2(b), mean values with different letters are significantly different $(p<0.05)$. CK: control group; BSA: BSA/ $\mathrm{NaOH}$ complex solution; PA: PA/BSA complex solution; "F" free phenolic fraction; "E" esterified phenolic fraction; "IB" insoluble-bound phenolic fraction. 
and $26.17 \pm 2.05 \mu \mathrm{moL} / \mathrm{g}$ of protein in UHP-treated fruit group, both of which were about $40 \%$ lower than that of the PA group $(p<0.05)$. However, UHP treatment did not significantly enhance the inhibitory effects of those three phenolic fractions on TG deposition in PA-induced HepG2 cells $(p>0.05)$.

Caffeic acid was the most abundant phenolic compound in both of UHP-treated and nontreated OPF [19]. Therefore, the inhibitory effect of caffeic acid on TG deposition was also clarified. In contrast with the PA group, the caffeic acid administration also significantly lowered TG content by approximately $30.45 \%(p<0.05)$. This result indicated that caffeic acid can effectively attenuate hepatic lipid accumulation, which was in accordance with the previous research: it was proved that the TG clearance was over $50 \%$ in oil acid induced HepG2 cells with the treatment of $40 \mu \mathrm{g} / \mathrm{mL}$ of caffeic acid [22]. Moreover, a previous study found that caffeic acid moiety in chlorogenic acid and its analogues were supposed to play a key role in the lipid-lowering activities of those compounds [23]. Therefore, the suppression effects of OPF phenolic fractions on hepatic fat accumulation probably attributed to caffeic acid and/or compounds with a similar structure.

\subsection{Phenolic Fractions and Caffeic Acid Reduced PA-Induced} ROS Generation. ROS was a cluster of high energy charged free radicals that are generally supposed to be responsible for many biological dysfunctions, such as inflammation, cells apoptosis, and MMP disruption [24, 25]. A previous study has verified that PA administration would lead to oxidative stress by producing lipid peroxides, accompanied by an increase of ROS level [26]. As presented in Figure 2(a), it was obvious that the fluorescence intensity of the PA group moved toward the right by comparison with that of the control group and BSA group (Figure 2(a)). And the ROS level in the PA group was $191.53 \%$, which was almost two times over that of the CK group or BSA group $(p<0.05$, Figure 2(b)), indicating serious oxidative stress under PA induction. When compared with the PA treatment, the fluorescence peak of each OPF phenolic fraction treatment gradually moved toward the left (Figure 2(a)), and the ROS generation of each treatment was correspondingly decreased $(p<0.05$, Figure 2(b)). Specifically, the ROS clearance effects of F, E, and IB fractions in nontreated fruits group were gradually increased, which reduced the ROS content to $174.14 \%, 156.65 \%$, and $139.25 \%$ respectively. The ROS inhibition trend of three phenolic fractions in UHP-treated fruit was similar to that in nontreated fruit. The scavenging effect of $\mathrm{F}$ fraction was weaker than the other two fractions, decreased the ROS amount to $166.86 \%$, and has no significant difference with the corresponding part in nontreated fruits $(p>0.05)$. The E and IB fractions declined the ROS amount to $142.72 \%$ and $127.79 \%$, respectively, both of which were significantly lower than the counterpart in nontreated fruits $(p<0.05)$. Generally, the UHP treatment significantly enhanced the ROS clearance capacity of OPF in PA-induced HepG2 cells, which was consistent with the previous result observed in $\mathrm{H}_{2} \mathrm{O}_{2}$-induced HepG2 cells [19], but no matter
UHP treated or not, the ROS production of IB fraction was the lowest and was followed by the E fraction.

As one of the major phenolic compounds in OPF, caffeic acid administration significantly attenuated the PA-induced ROS generation by $21.8 \%(p<0.05$, Figure $2(b))$, which was markedly better than that of the $\mathrm{F}$ fraction treatment, but had significantly lesser effect than that of the IB fraction treatment $(p<0.05)$. Besides caffeic acid, some other phenolics in the IB fraction may also contribute to its ROS inhibitory capacity. For example, catechin and protocatechuic acid were also the primary phenolic compounds detected in an insoluble-bound fraction [19]. Wong et al. [27] reported that $0.3 \mathrm{mM}$ catechin reduced the amount of ROS by $38 \%$ in PA-induced astrocytes. In another study, $10 \mu \mathrm{M}$ catechin can decrease 54\% ROS generation in PAinduced HepG2 cells [28]. Moreover, it also found that protocatechuic acid could significantly attenuate ROS generation by activating endogenous antioxidant enzymes [29].

3.3. Phenolic Fractions and Caffeic Acid Prevented PA-Induced Cell Apoptosis. The excessive ROS not only leads to oxidative damage but also impairs cells and thereby induces cell apoptosis, which partly accounts for the autoimmune disorders and cancers [30-32]. As OPF phenolic fractions exhibited significant ROS scavenge ability, the inhibition effects of them on PA-induced apoptosis were further evaluated by flow cytometry assay. As shown in Figure 3(a), the apoptotic cells appear in two parts of each panel of Figure 3(a): the cells in late apoptosis are presented in the upper right gate, and the early apoptosis cells are showed in the lower right gate [33]. Based on the results in Figure 3(a), the corresponding cell apoptotic proportion was calculated in Figure 3(b). As shown in Figure 3(b), the cell apoptotic rate of the PA group was about $43.06 \%$, which was significantly higher than that of the CK group or the BSA group $(p<0.05)$. The amounts of normal cells in the CK group was $92.42 \%$ and the BSA group was $88.69 \%$; the slight decrease in the survival rate of the BSA group may be due to the introduction of $\mathrm{NaOH}$ with the $\mathrm{BSA} / \mathrm{NaOH}$ complex solution. With the treatment of the OPF phenolic fractions, the PAinduced cell apoptosis was attenuated. Regardless of UHP treatment, the IB fraction exhibited the best protective effect against the PA-induced cell apoptosis, followed by the $\mathrm{E}$ fraction $(p<0.05)$, while the $\mathrm{F}$ fraction from OPF showed the weakest protective activity $(p<0.05)$. When OPF was pretreated with UHP, the IB fraction exerted significantly better protection against cell apoptosis than the counterpart of OPF without UHP treatment $(p<0.05)$. However, UHP treatment did not significantly enhance the protective activities of the $\mathrm{F}$ and $\mathrm{E}$ fractions from OPF. These results indicated that the inhibitory effect of IB fraction on cell apoptosis was upgraded after UHP treatment, which is probably due to its higher phenolic content [19] and the better antioxidant ability (Figure 2).

The result of caffeic acid inhibiting the PA-induced cell apoptosis was also determined (Figure 3). In contrast with PA treatment, caffeic acid significantly inhibited cell 

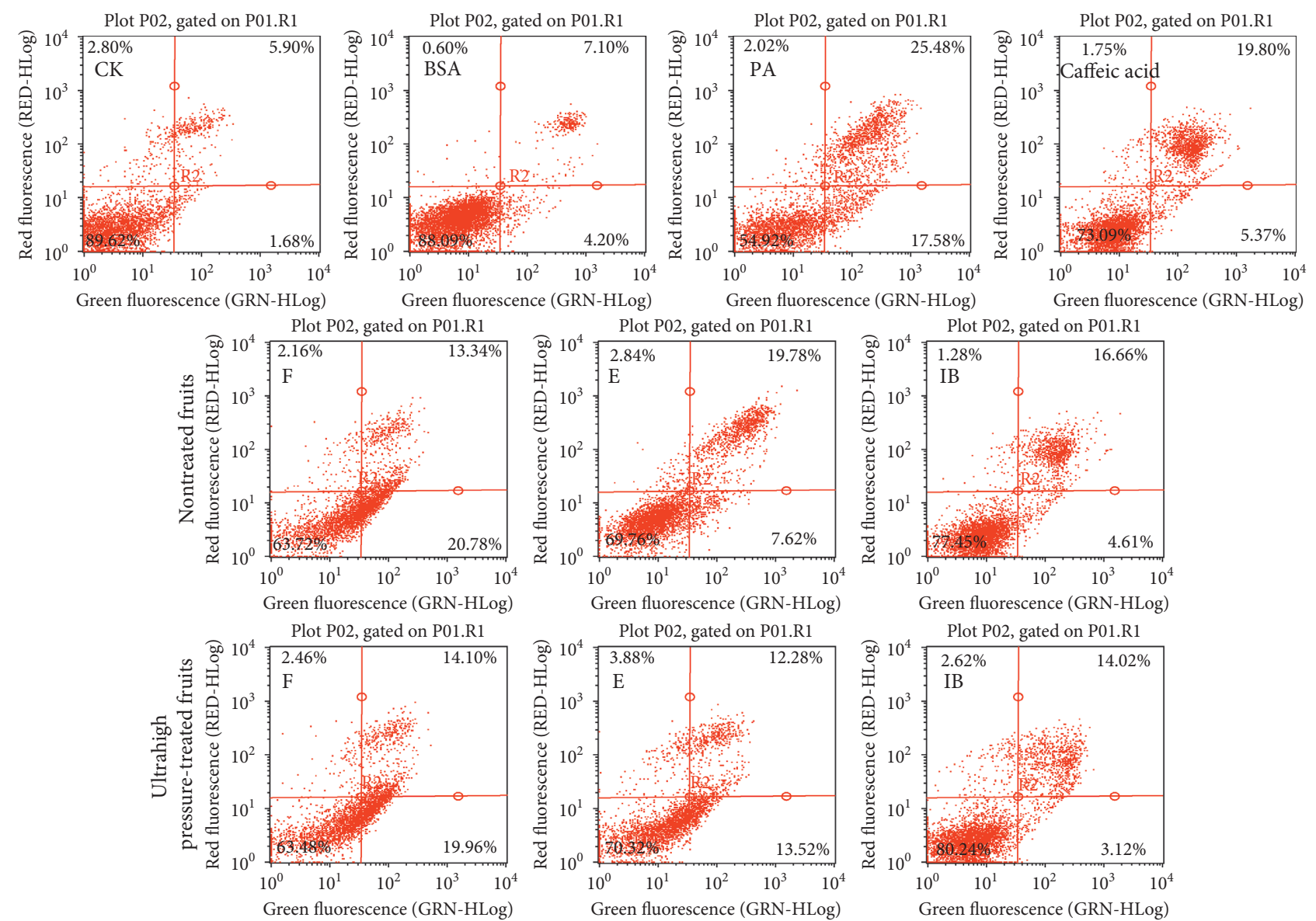

(a)

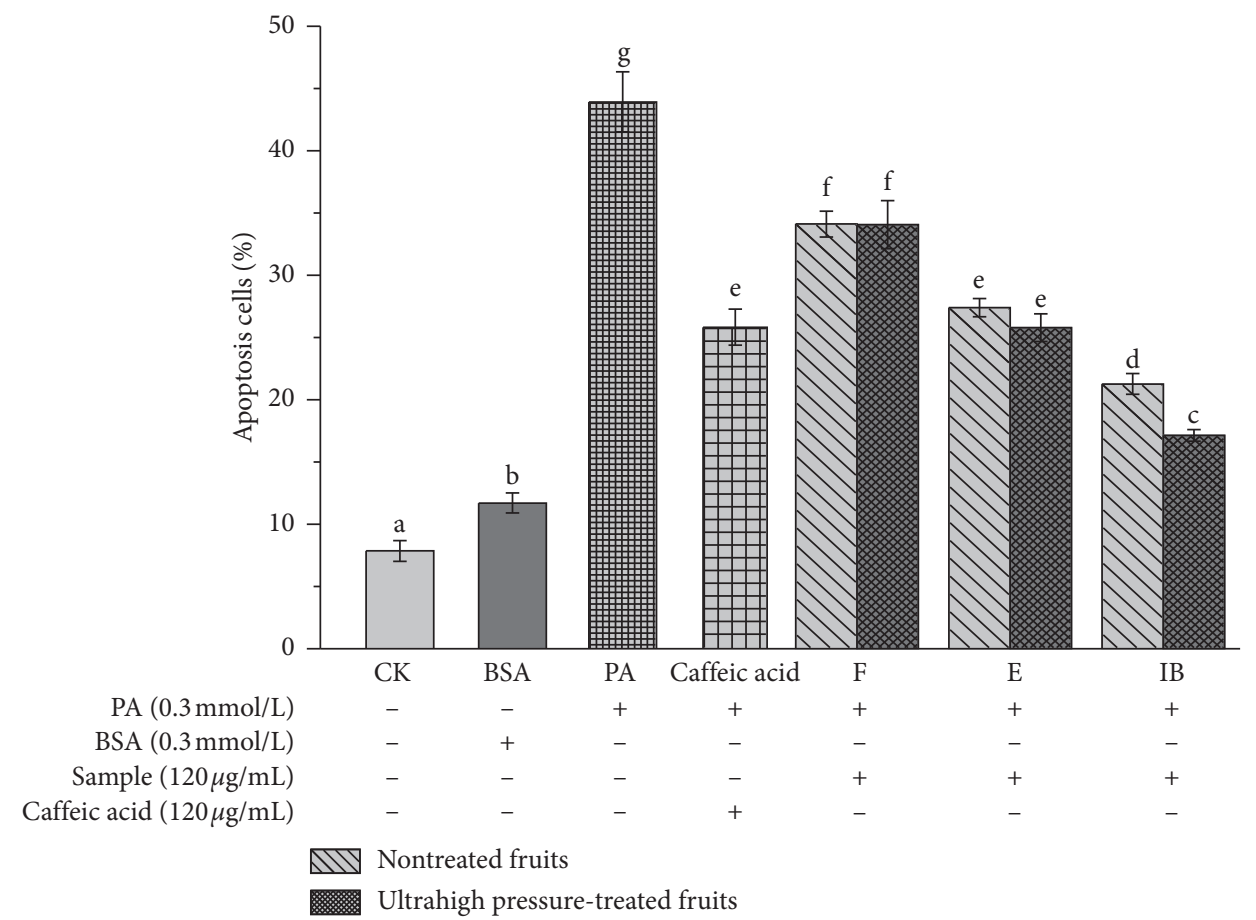

(b)

Figure 3: The inhibitory effects of phenolic fractions from oil palm fruits with or without ultrahigh pressure treatment and caffeic acid on PA-induced cell apoptosis: (a) flow cytometry analysis, (b) the relative apoptosis rate of different groups. Data are expressed as mean \pm SD $(n=3)$. In Figure 3(b), mean values with different letters are significantly different $(p<0.05)$. CK: control group; BSA: BSA/NaOH complex solution; PA: PA/BSA complex solution; "F" free phenolic fraction; "E" esterified phenolic fraction; "IB" insoluble-bound phenolic fraction. 
apoptosis by $41.55 \%(p<0.05)$, indicating caffeic acid might be the main functional compound in OPF to inhibit PAinduced cell apoptosis. Besides, a previous study reported that kaempferol, also detected in IB fraction, could increase the cell viability from $68 \%$ to $98 \%$ in the PA-induced pancreatic $\beta$-cell [10]. Moreover, it is worth noting that the protective effect of the IB fraction, regardless of UHP treatment, was significantly better than that of caffeic acid. Therefore, those results indicated that other phenolic compounds in OPF may also contribute to the prevention against PA-induced cell apoptosis, and those phenolic compounds may interact with each other to exert a better protective activity.

3.4. Phenolic Fractions and Caffeic Acid Ameliorated PAInduced MMP Depolarization. The mitochondrion is a crucial organelle, that not only undertakes the energy regulation but also controls the balance of ROS generation [9]. Under the condition of lipotoxicity, the massive ROS could rapidly diffuse to the mitochondrion, lead to MMP depolarization, and thereby mediate cell apoptosis [29, 34]. Therefore, bioactive compounds with ROS scavenge ability may inhibit cell apoptosis through mitochondria protection [35]. In this study, the MMP of each group was monitored by a flow cytometer with the probe of JC-1. JC-1 could penetrate into mitochondria and show different fluorescence to reveal the MMP. Briefly, the red fluorescence was triggered when the MMP was high, while the green fluorescence was produced when the MMP decreased [36]. Based on these, the mitochondrial membrane depolarization level could be calculated as the ratio of the red fluorescence intensity to the green fluorescence intensity.

As shown in Figure 4, the CK group has strong red fluorescence intensity and a stable MMP with a red-to-green ratio of about 8.5 , and this ratio in the BSA group was about 6.8. The minor decline of the ratio in the BSA group may be due to the effect of $\mathrm{NaOH}$ in $\mathrm{BSA} / \mathrm{NaOH}$ complex solution. When treated with PA, the red fluorescence intensity was obviously declined (Figure 4(a)), and the red-to-green ratio of the PA group was significantly decreased in comparison with that of the CK group or the BSA group $(p<0.05$, Figure 4(b)), indicating that PA treatment decreased MMP and thereby caused a serious mitochondrial membrane depolarization. Pretreatment of OPF phenolic fractions showed a protective effect on PA-induced mitochondria damage. As demonstrated in Figure 4, the red-to-green ratios of three phenolic fractions in nontreated fruits were about 2.54, 2.93, and 3.79, respectively. As for UHP-treated fruits, the red-to-green values of the corresponding phenolic fractions were $2.57,3.35$, and 4.95 . It was obvious that, no matter UHP treatment or not, all the three phenolic fractions of OPF can effectively alleviate the depolarized mitochondrial membrane potential caused by PA, and the effect of the IB fraction was the best $(p<0.05)$. Meanwhile, the results clearly showed that UHP treatment significantly enhanced the protective effect of the IB fraction against PA-induced the depolarization of MMP $(p<0.05)$. Therefore, the lipotoxicity preventive effect of OPF phenolic fractions may be partly achieved by protecting mitochondrial function. The ameliorative effect of phenolic compounds on mitochondrial membrane depolarization has been previously reported. Jin et al. [6] manifested that Chinese propolis treatment can rescue the MMP decrease induced by PA in HepG2 cells, and composition analysis showed that Chinese propolis contained many phenolic compounds, including ferulic acid, caffeic acid, and kaempferol, and these phenolics has also been detected in the different phenolic fractions from OPF [19]. Moreover, it is found that catechin pretreatment could almost protect the MMP of HepG2 cells from the influence of PA treatment [28]. In our previous study, caffeic acid was found to be the most abundant phenolic compound in all the OPF phenolic fractions, while the information about its effect on MMP maintenance of the lipotoxicity condition has not been investigated. Result in the current study showed that the red fluorescence intensity of caffeic acid treatment was significantly higher than that of the PA group with the mean value of the red-to-green ratio rising from 3.10 to 3.58 , indicating that the mitochondrial membrane depolarization was significantly improved by caffeic acid treatment $(p<0.05$, Figure 4$)$. This result may provide evidence that caffeic acid was one of the bioactive compounds contributing to the MMP protection of the different phenolic fractions from OPF.

\subsection{Preventive Mechanisms of the IB Fractions and Caffeic} Acid in PA-Induced Lipotoxicity. According to the abovementioned results, regardless of UHP treatment, IB phenolic fraction showed the best effects on TG clearance, ROS reduction, inhibition of cells apoptosis, and MMP maintenance among the three OPF phenolic fractions. Therefore, the underlying mechanisms of IB phenolic fractions from OPF with or without UHP treatment and caffeic acid on alleviating PA-induced lipotoxicity in HepG2 cells were comparatively investigated by Western blot analysis.

Massive ROS would be generated with PA induction, which may lead to an inflammatory response and finally result in cell apoptosis [11]. COX-2 and iNOS are two characteristic inflammation cytokines that expression levels sensitively increase with excessive ROS production [37]. Under the stimulation of inflammation cytokines, the P38 protein would be phosphorylated, which may activate the P38-MAPK pathway to regulate physiological and pathological processes, such as inflammation, cell growth, and apoptosis $[12,13]$. As shown in Figure 5, in contrast with the CK group, the expressions of P-P38, COX-2, and iNOS proteins in the PA group increased significantly $(p<0.05)$. And the expression levels of those proteins in the PA group were about four times, seven times, and three times of those in the CK group, respectively. However, the administration of IB phenolic fractions, regardless of UHP treatment, significantly inhibited the expressions of those proteins in comparison with the counterpart of the PA group $(p<0.05)$. Besides, the IB fraction from the UHP-treated OPF exhibited better effect in inhibiting COX-2 expression than that of the IB fraction from the nontreated OPF (Figure 5, $p<0.05$ ). The antioxidant ability of the IB fraction increased 

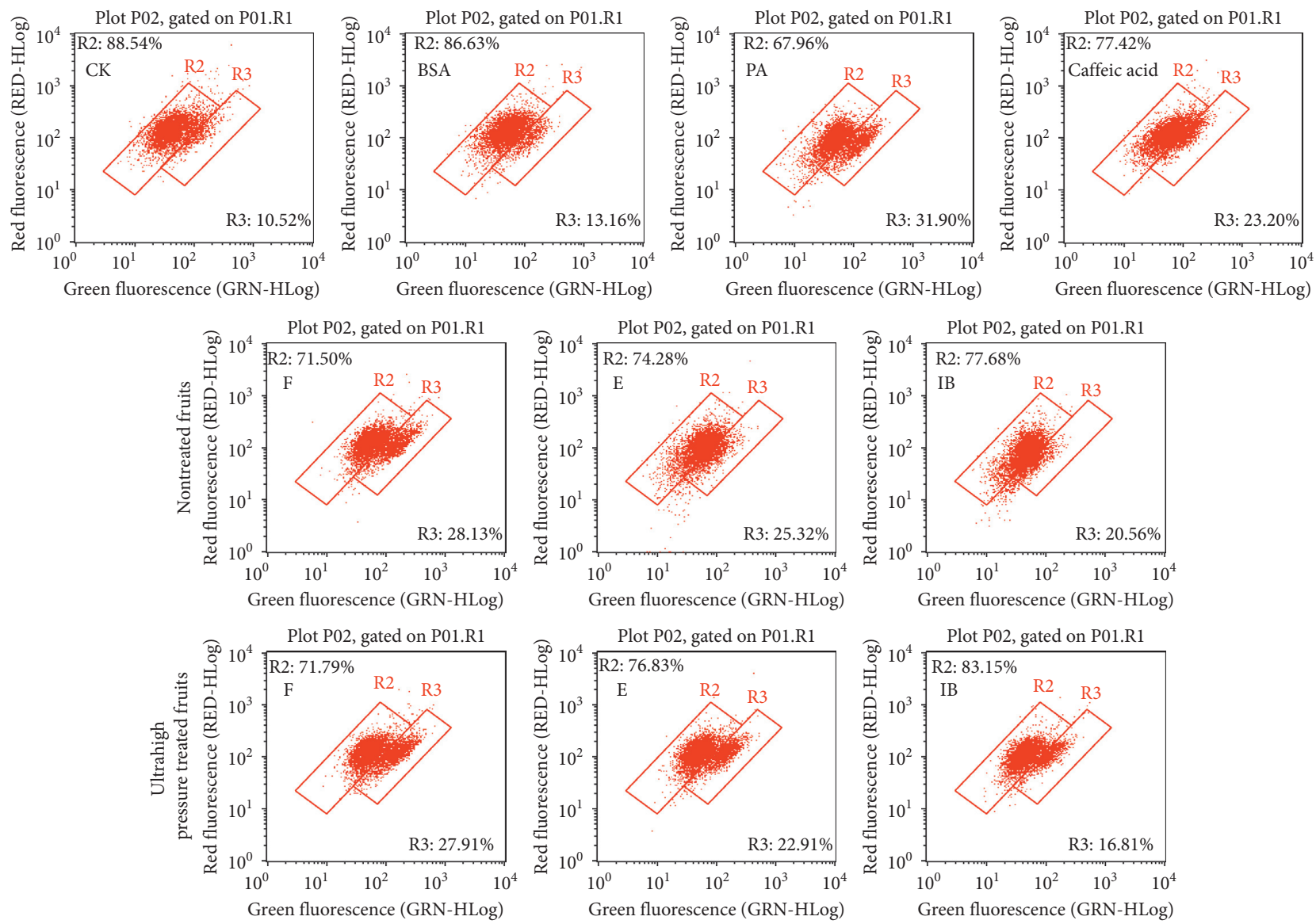

(a)

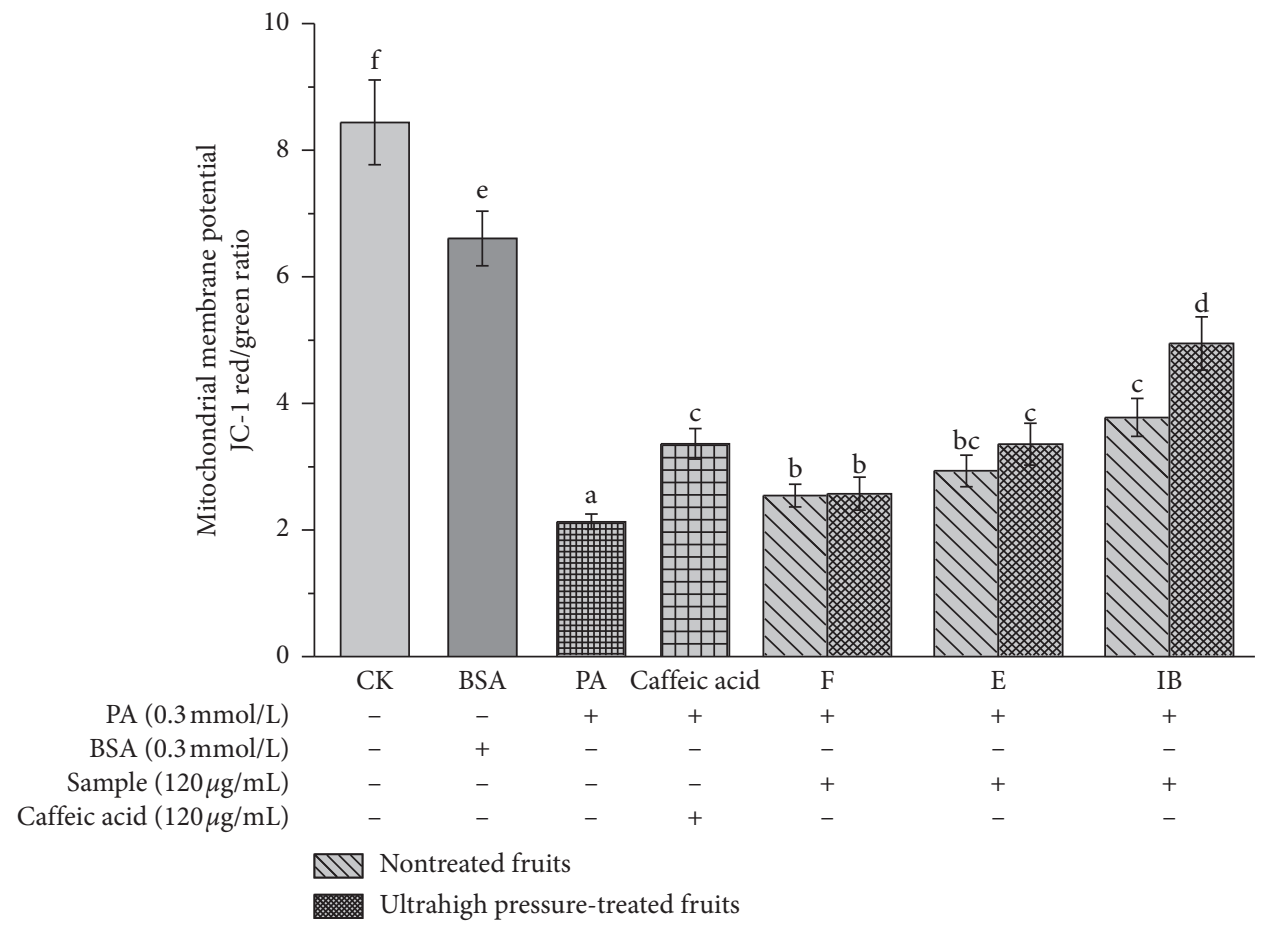

(b)

FIGURE 4: The inhibitory effects of phenolic fractions from oil palm fruits with or without ultrahigh pressure treatment and caffeic acid on PA-induced MMP depolarization: (a) flow cytometry analysis, (b) the ratio of red fluorescence to green fluorescence of different groups. Data are expressed as mean \pm SD $(n=3)$. In Figure 4(b), mean values with different letters are significantly different $(p<0.05)$. CK: control group; BSA: BSA/NaOH complex solution; PA: PA/BSA complex solution; MMP: mitochondrial membrane potential; "F" free phenolic fraction; "E" esterified phenolic fraction; "IB" insoluble-bound phenolic fraction. 


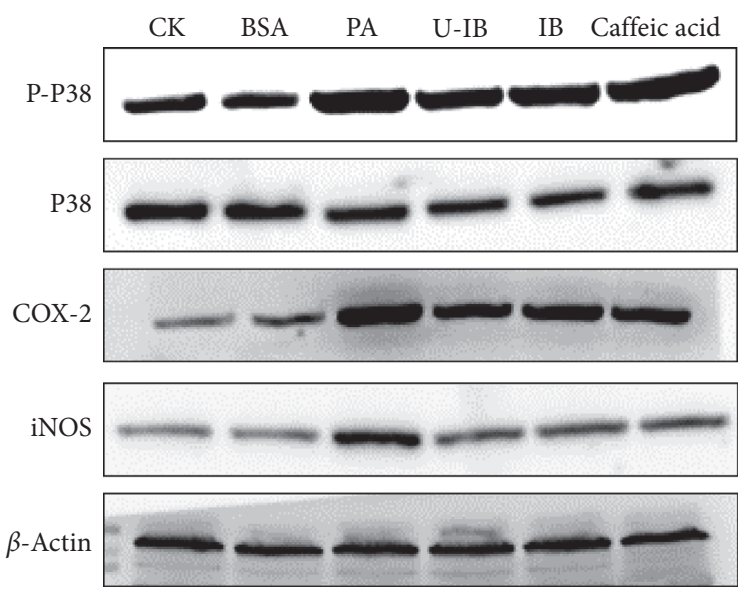

(a)

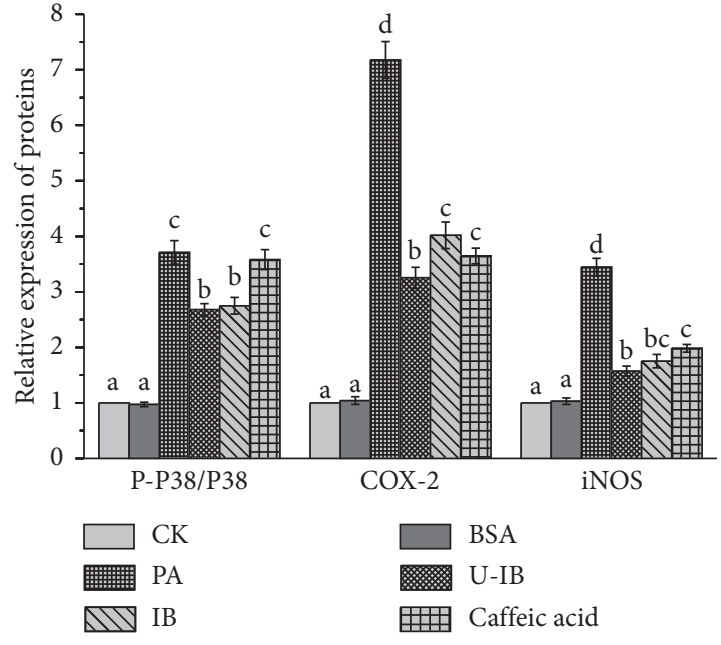

(b)

FIGURE 5: Effects of insoluble-bound fraction and caffeic acid on the protein expression levels of P38, COX-2, and iNOS (a) and the relative protein expression levels (b). Protein expression levels were normalized with $\beta$-actin, according to grayscale. Data are expressed as mean \pm SD $(n=3)$. In Figure 5(b), mean values with different letters are significantly different $(p<0.05)$. CK: control group; BSA: BSA/ $\mathrm{NaOH}$ complex solution; PA, PA/BSA complex solution; P38, P38 mitogen activated protein kinases; COX-2: cyclooxygenase-2; iNOS: inducible nitric oxide synthase; IB: insoluble-bound phenolic fraction; U-IB: UHP-treated insoluble-bound phenolic fraction.

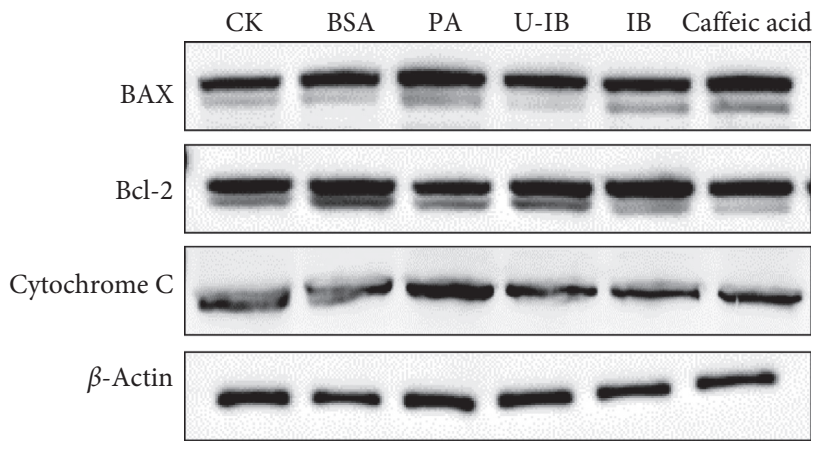

(a)

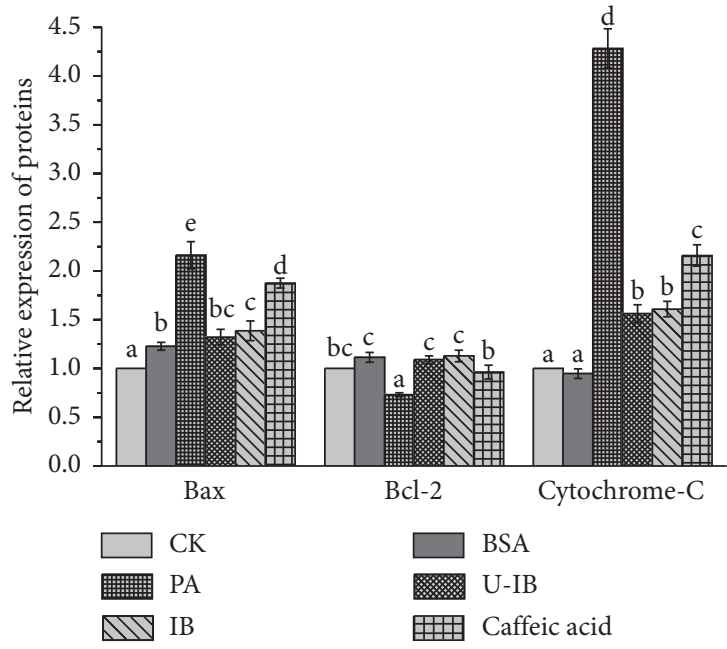

(b)

FIGURE 6: (a) Effects of insoluble-bound fraction and caffeic acid on the protein expression levels of Bax, Bcl-2, and cytochrome C, (b) the relative protein expression levels. Protein expression levels were normalized with $\beta$-actin according to grayscale. Data are expressed as mean \pm SD $(n=3)$. In Figure 6(b), mean values with different letters are significantly different $(p<0.05)$. CK: control group; BSA: BSA/ $\mathrm{NaOH}$ complex solution; PA: PA/BSA complex solution; Bax: Bcl-2-associated X protein; Bcl-2: B cell lymphoma-2; IB: insoluble-bound phenolic fraction; U-IB: UHP-treated insoluble-bound phenolic fraction.

significantly [19], and the generation of ROS was remarkably suppressed (Figure 2(b)) after the UHP treatment, which may further lower the oxidative stress of cells to cause lesser expression of inflammatory cytokines and thereby exhibit better cell protection. Caffeic acid administration significantly inhibited the expressions of COX-2 and iNOS proteins when compared with that of the corresponding protein in the PA group $(p<0.05)$. However, caffeic acid did not remarkably reduce the expression ratio of $\mathrm{P}-\mathrm{P} 38 / \mathrm{P} 38$ $(p>0.05)$.
In addition to inflammatory pathways, the mitochondrial pathway is also mediated by ROS and may lead to cell apoptosis $[11,38]$. Bax and Bcl-2 were apoptotic related proteins of the Bcl-2 family, which played an important role in the mitochondrial pathway. A phenolic compound like naringin was proved to inhibit cell apoptosis by regulating the $\mathrm{Bax} / \mathrm{Bcl}-2$ pathway [39]. In the PA treatment group of the present study, the expression level of Bax was increased significantly when compared with that in the CK group or BSA group (Figure 6), which indicated the proapoptosis protein was activated and 
would interact with mitochondrial membrane to decline MMP. Meanwhile, the Bcl-2 expression was declined remarkably by the PA treatment (Figure 6), which indicated that the bioactivity of this antiapoptosis protein may reduce. With the effects of the PA treatment on Bax and Bcl-2, the MMP would be disrupted and the mitochondrial permeability might be damaged, and thereby finally stimulate cell apoptosis, which could be proved as the increased expression level of cytochrome $\mathrm{C}$ in this study. When cells were incubated with IB fraction, regardless of UHP treatment, the expression of Bax was significantly inhibited while the expression of Bcl-2 was markedly promoted in contrast with the PA group (Figure 6, $p<0.05$ ), which provide possible pathways for IB fraction administration to achieve a higher MMP, decrease mitochondrial membrane depolarization level, and prevent the PA induced cell apoptosis. However, UHP treatment did not significantly enhance the effects of the IB phenolic fraction on the expressions of the Bax, Bcl-2, and cytochrome C proteins $(p>0.05)$. Caffeic acid also significantly inhibited the expressions of the Bax and cytochrome $\mathrm{C}$ proteins and upgraded the $\mathrm{Bcl}-2$ expression when compared with that of the counterpart in the PA group $(p<0.05)$. However, the effect of caffeic acid was weaker than those of the IB phenolic fractions with or without UHP treatment, indicating some other phenolic compounds in the IB phenolic fraction may also contribute to the antiapoptosis, which needs to be proved by further experiments.

\section{Conclusions}

The results of this study revealed that the OPF phenolic fractions could prevent the PA induced lipotoxicity. Among the three phenolic fractions, the IB phenolic fraction showed the best effect to inhibit TG accumulation, reduce intracellular ROS, decrease the apoptotic rate, and prevent MMP depolarization. After the UHP treatment, the protective effect of the IB phenolic fraction was increased. Inhibition of inflammation by downregulating the expression of P-P38, COX-2, and iNOS, and suppression of mitochondrial mediated apoptosis by downregulating Bax and cytochrome $\mathrm{C}$ and upregulating $\mathrm{Bcl}-2$ might be the potential mechanism pathway for the IB phenolic fraction to achieve the lipotoxicity preventive effect. Besides, as the most abundant phenolic compound in OPF phenolic fractions, caffeic acid exhibited similar effects as the IB fraction, and probably achieved such effect through the same pathway. Therefore, the OPF phenolic fractions, especially the IB phenolic fraction, might be utilized as natural materials for the development of antilipotoxicity functional food. The UHP treatment might be the potential method to increase this bioactivity of OPF.

\section{Data Availability}

All data included in this study are available upon request by contacting the corresponding author.

\section{Conflicts of Interest}

There are no conflicts of interest regarding the publication of this article.

\section{Authors' Contributions}

Xiaojing Liu and Ou Wang contributed equally to this work.

\section{Acknowledgments}

This work was financially supported by the National Natural Science Foundation of China (grant no. 31960477) and Yunnan Major Science and Technology Project (2019ZF010).

\section{References}

[1] J. W. Yun, "Possible anti-obesity therapeutics from nature-a review," Phytochemistry, vol. 71, no. 14-15, pp. 1625-1641, 2010.

[2] V. Pardo, Á. González-Rodríguez, J. Muntané, S. C. Kozma, and Á. Valverde, "Role of hepatocyte S6K1 in palmitic acidinduced endoplasmic reticulum stress, lipotoxicity, insulin resistance and in oleic acid-induced protection," Food and Chemical Toxicology, vol. 80, pp. 298-309, 2015.

[3] H. Nishi, T. Higashihara, and R. Inagi, "Lipotoxicity in kidney, heart, and skeletal muscle dysfunction," Nutrients, vol. 11, no. 7, pp. 1664-1681, 2019.

[4] M. Izdebska, I. Piątkowska-Chmiel, A. Korolczuk et al., "The beneficial effects of resveratrol on steatosis and mitochondrial oxidative stress in HepG2 cells," Canadian Journal of Physiology and Pharmacology, vol. 95, no. 12, pp. 1442-1453, 2017.

[5] C. Yang, W. Bazer, and G. Song, "Down-regulation of stearoyl-CoA desaturase-1 increases susceptibility to palmitic-acid-induced lipotoxicity in human trophoblast cells," The Journal of Nutritional Biochemistry, vol. 54, pp. 35-47, 2018.

[6] X.-L. Jin, K. Wang, Q.-Q. Li et al., "Antioxidant and antiinflammatory effects of Chinese propolis during palmitic acid-induced lipotoxicity in cultured hepatocytes," Journal of Functional Foods, vol. 34, pp. 216-223, 2017.

[7] A. Ayala, M. F. Muñoz, and S. Argüelles, "Lipid peroxidation: production, metabolism, and signaling mechanisms of malondialdehyde and 4-hydroxy-2-nonenal," Oxidative Medicine and Cellular Longevity, vol. 2014, Article ID 360438, 31 pages, 2014.

[8] P. Ye, R. Gan, H. Li et al., "Absorption, metabolism, and bioactivity of vitexin: recent advances in understanding the efficacy of an important nutraceutical," Critical Reviews in Food Science and Nutrition, pp. 1-16, 2020, In press.

[9] D. Hu, X. Yang, J. Xie, C. Sun, X. Zheng, and W. Chen, "Systematic evaluation of phenolic compounds and protective capacity of a new mulberry cultivar J33 against palmitic acidinduced lipotoxicity using a simulated digestion method," Food Chemistry, vol. 258, pp. 43-50, 2018.

[10] R. Varshney, S. Gupta, and P. Roy, "Cytoprotective effect of kaempferol against palmitic acid-induced pancreatic $\beta$-cell death through modulation of autophagy via AMPK/mTOR signaling pathway," Molecular and Cellular Endocrinology, vol. 448, pp. 1-20, 2017.

[11] J. Wang, L. Yuan, H. Xiao, C. Xiao, Y. Wang, and X. Liu, "Momordin Ic induces HepG2 cell apoptosis through MAPK and PI3K/Akt-mediated mitochondrial pathways," Apoptosis, vol. 18, no. 6, pp. 751-765, 2013.

[12] E. M. Galan-Moya, A. Miguel, and M. L. Valero, "Balance between MKK6 and MKK3 mediates p38 MAPK associated resistance to cisplatin in NSCLC," PloS One, vol. 6, no. 12, Article ID e28406, 2011. 
[13] S.-H. Lee, C.-H. Kwak, S.-K. Lee et al., "Anti-inflammatory effect of ascochlorin in LPS-stimulated RAW 264.7 macrophage cells is accompanied with the down-regulation of iNOS, COX-2 and proinflammatory cytokines through NF- $\kappa \mathrm{B}$, ERK1/2, and p38 signaling pathway," Journal of Cellular Biochemistry, vol. 117, no. 4, pp. 978-987, 2016.

[14] L. Zhao, S. Li, L. Zhao, Y. Zhu, and T. Hao, "Antioxidant activities and major bioactive components of consecutive extracts from blue honeysuckle (Lonicera caerulea L.) cultivated in China," Journal of Food Biochemistry, vol. 39, no. 6, pp. 653-662, 2015.

[15] M. Bian, X. Chen, C. Zhang et al., "Magnesium isoglycyrrhizinate promotes the activated hepatic stellate cells apoptosis via endoplasmic reticulum stress and ameliorates fibrogenesisin vitroandin vivo," Biofactors, vol. 43, no. 6, pp. 836-846, 2017.

[16] J. T. Opferman and A. Kothari, "Anti-apoptotic BCL-2 family members in development," Cell Death \& Differentiation, vol. 25, no. 1, pp. 37-45, 2018.

[17] Y. Jiong and Y. Shukun, "JNK-Bcl-2/Bcl-xL-Bax/Bak pathway mediates the crosstalk between matrine-induced autophagy and apoptosis via interplay with beclin 1," International Journal of Molecular Sciences, vol. 16, no. 10, pp. 25744-25758, 2015.

[18] Y. Youn and Y.-S. Kim, "Inhibitory effects of Citrus unshiu pericarpium extracts on palmitate-induced lipotoxicity in HepG2 cells," Food Science and Biotechnology, vol. 25, no. 6, pp. 1709-1717, 2016.

[19] J. Zhou, Y. Ma, Y. Jia, M. Pang, G. Cheng, and S. Cai, "Phenolic profiles, antioxidant activities and cytoprotective effects of different phenolic fractions from oil palm (Elaeis guineensis Jacq.) fruits treated by ultra-high pressure," Food Chemistry, vol. 288, pp. 68-77, 2019.

[20] S. P. Cousin, S. R. Hügl, C. E. Wrede, H. Kajio, M. G. Myers, and C. J. Rhodes, "Free fatty acid-induced inhibition of glucose and insulin-like growth factor I-induced deoxyribonucleic acid synthesis in the pancreatic $\beta$-cell line INS11," Endocrinology, vol. 142, no. 1, pp. 229-240, 2001.

[21] Y. Zang, L. Fan, J. Chen, R. Huang, and H. Qin, "Improvement of lipid and glucose metabolism by capsiate in palmitic acid-treated HepG2 cells via activation of the AMPK/SIRT1 signaling pathway," Journal of Agricultural and Food Chemistry, vol. 66, no. 26, pp. 6772-6781, 2018.

[22] Y. Liu, D. Wang, D. Zhang et al., "Inhibitory effect of blueberry polyphenolic compounds on oleic acid-induced hepatic steatosis in vitro," Journal of Agricultural and Food Chemistry, vol. 59, no. 22, pp. 12254-12263, 2011.

[23] X. Cao, C. Wu, Y. Tian, and P. Guo, "The caffeic acid moiety plays an essential role in attenuating lipid accumulation by chlorogenic acid and its analogues," RSC Advances, vol. 9, no. 22, pp. 12247-12254, 2019.

[24] D. Lin, M. Xiao, J. Zhao et al., "An overview of plant phenolic compounds and their importance in human nutrition and management of type 2 diabetes," Molecules, vol. 21, no. 10, pp. 1374-1393, 2016.

[25] H.-M. Shen, C.-F. Yang, W.-X. Ding, J. Liu, and C.-N. Ong, "Superoxide radical-initiated apoptotic signalling pathway in selenite-treated HepG2 cells: mitochondria serve as the main target," Free Radical Biology and Medicine, vol. 30, no. 1, pp. 9-21, 2001.

[26] W. Gehrmann, W. Würdemann, T. Plötz, A. Jörns, S. Lenzen, and M. Elsner, "Antagonism between saturated and unsaturated fatty acids in ROS mediated lipotoxicity in rat insulinproducing cells," Cellular Physiology and Biochemistry, vol. 36, no. 3, pp. 852-865, 2015.
[27] K.-L. Wong, Y.-R. Wu, K.-S. Cheng et al., "Palmitic acidinduced lipotoxicity and protection by $(+)$-catechin in rat cortical astrocytes," Pharmacological Reports, vol. 66, no. 6, pp. 1106-1113, 2014.

[28] H. Rafiei, K. Omidian, and B. Bandy, "Protection by different classes of dietary polyphenols against palmitic acid-induced steatosis, nitro-oxidative stress and endoplasmic reticulum stress in HepG2 hepatocytes," Journal of Functional Foods, vol. 44, no. 1, pp. 173-182, 2018.

[29] L. Han, Q. Yang, W. Ma, J. Li, L. Qu, and M. Wang, "Protocatechuic acid ameliorated palmitic-acid-induced oxidative damage in endothelial cells through activating endogenous antioxidant enzymes via an adenosine-monophosphate-activated-protein-kinase-dependent pathway," Journal of Agricultural and Food Chemistry, vol. 66, no. 40, pp. 10400-10409, 2018.

[30] A. Ortiz-Rodriguez, E. Acaz-Fonseca, P. Boya, M. A. Arevalo, and L. M. Garcia-Segura, "Lipotoxic effects of palmitic acid on astrocytes are associated with autophagy impairment," Molecular Neurobiology, vol. 56, no. 3, pp. 1665-1680, 2019.

[31] D. Smolensky, D. Rhodes, D. S. Mcvey et al., "Highpolyphenol sorghum bran extract inhibits cancer cell growth through ROS induction, cell cycle arrest, and apoptosis," Journal of Medicinal Food, vol. 21, no. 10, pp. 990-998, 2018.

[32] Y. Zhuang, Q. Ma, Y. Guo, and L. Sun, "Protective effects of rambutan (Nephelium lappaceum) peel phenolics on $\mathrm{H} 2 \mathrm{O} 2$ -induced oxidative damages in HepG2 cells and d-galactoseinduced aging mice," Food and Chemical Toxicology, vol. 108, pp. 554-562, 2017.

[33] L. Wang, L. Ding, Z. Yu, T. Zhang, S. Ma, and J. Liu, "Intracellular ROS scavenging and antioxidant enzyme regulating capacities of corn gluten meal-derived antioxidant peptides in HepG2 cells," Food Research International, vol. 90, pp. 33-41, 2016.

[34] B. L. Tan, M. E. Norhaizan, and L. C. Chan, "ROS-mediated mitochondrial pathway is required for Manilkara zapota (L.) P. Royen leaf methanol extract inducing apoptosis in the modulation of caspase activation and EGFR/NF- $\kappa \mathrm{B}$ activities of HeLa human cervical cancer cells," Evidence-Based Complementary and Alternative Medicine, vol. 2018, Article ID 6578648, 19 pages, 2018.

[35] J. Xiang, F. B. Apea-Bah, V. U. Ndolo, M. C. Katundu, and T. Beta, "Profile of phenolic compounds and antioxidant activity of finger millet varieties," Food Chemistry, vol. 275, pp. 361-368, 2019.

[36] S. Paul and R. Kundu, "ROS mediated DNA damage and induction of apoptosis in cervical cancer cells by Heliotropium indicum L," Journal of Applied Pharmaceutical Science, vol. 8, no. 8, pp. 92-106, 2018.

[37] T. Pauloin, M. Dutot, F. Joly, J. M. Warnet, and P. Rat, "High molecular weight hyaluronan decreases UVB-induced apoptosis and inflammation in human epithelial corneal cells," Molecular Vision, vol. 15, pp. 577-583, 2009.

[38] A. P. Kaur and S. Agrawal, "A review of the molecular mechanism of apoptosis and its role in pathological conditions," International Journal of Pharma and Bio Sciences, vol. 10, no. 2, pp. 124-131, 2019.

[39] J. Cui, G. Wang, A. D. Kandhare, A. A. Mukherjee-Kandhare, and S. L. Bodhankar, "Neuroprotective effect of naringin, a flavone glycoside in quinolinic acid-induced neurotoxicity: possible role of PPAR- $\gamma$, Bax/Bcl-2, and caspase-3," Food and Chemical Toxicology, vol. 121, pp. 95-108, 2018. 